\title{
El rol de la lengua en el proyecto histórico de los Comentarios reales del Inca Garcilaso
}

\section{The Role of the Language in the Historical Project in Inca Garcilaso's comentarios reales}

\section{Carlos Jesús Torres Astocóndor}

https://orcid.org/0000-0001-8676-5802

Pontificia Universidad Católica del Perú

Universidad Privada del Norte

PERÚ

cjtorres@pucp.pe

[Hipogrifo, (issn: 2328-1308), 8.2, 2020, pp. 805-816]

Recibido: 16-01-2020 / Aceptado: 24-09-2020

DOI: http://dx.doi.org/10.13035/H.2020.08.02.47

Resumen. El siguiente trabajo tiene como objetivo comprender la valoración del uso de la lengua en los Comentarios reales del Inca Garcilaso de la Vega. Para ello, analizo dos escenas en el texto. En primer lugar, busco leer la historia de Pedro Serrano en los Comentarios reales como "verdad poética". Ello me permitirá sostener que este relato estima la lengua como vía de comprensión del otro y como herramienta para establecer relaciones entre sujetos y construir identidades. En segundo lugar, enumero las funciones que cumplió la lengua general durante el gobierno de los Incas y de qué forma otorgó cohesión social a pesar de las diferencias culturales de los pueblos conquistados por el Imperio incaico. Ambos aspectos inciden en una conciencia idiomática en el Inca Garcilaso que trasciende las correcciones lingüística y apuesta por un plano identitario individual y colectivo.

Palabras claves. Inca Garcilaso de la Vega; conciencia idiomática; función de la lengua; verdad poética; Pedro Serrano.

Abstract. This work aims to understand the value of the use of language in the Comentarios reales by Inca Garcilaso de la Vega. For this purpose, two scenes in the text are analyzed. Firstly, I seek to read the story of Pedro Serrano in the Comentarios reales as «poetic truth». This will allow me to hold that this story considers language as a way of understanding the other and as a tool for establishing rela- 
tionships between subjects and developing identities. Secondly, I enumerate the functions that the general language fulfilled during the government of the Incas and how it provided social cohesion despite the cultural differences of the peoples conquered by the Inca empire. Both aspects have an impact on Inca Garcilaso's language awareness that transcends linguistic corrections and focuses on individual and collective identity.

Keywords. Inca Garcilaso de la Vega; Idiomatic awareness; Language function; Poetic truth; Pedro Serrano.

El siguiente trabajo analiza algunos capítulos de la primera parte de los Comentarios reales (1609) del Inca Garcilaso de la Vega para explicar que el uso de la lengua, en el Imperio incaico, se constituyó como el elemento cohesionador que buscó definir a una comunidad y otorgarle una identidad colectiva. Por un lado, a partir del análisis del origen del nombre del Perú con relación al cuento de Pedro Serrano, Garcilaso valora la función relacional que cumple la lengua en la comprensión del otro, lo que permite identificar y entablar una relación con este. Por otro lado, a través del análisis de la expansión geopolítica del Imperio incaico, la lengua sirvió para afianzar las relaciones sociales y entablar una correspondencia directa entre las autoridades mayores del Imperio (Incas o ministros) y el pueblo. En efecto, se puede rastrear funciones de tipo político, económico, pedagógico, social, cultural, bélico y religioso que cumplió el uso del quechua.

\section{INCOMUNICACIÓN LINGÜÍSTICA Y CONCIENCIA IDIOMÁTICA: UNA RELECTURA DEL CUENTO SOBRE PEDRO SERRANO}

El nombre del Perú, según el Inca Garcilaso, responde a una incomunicación lingüística entre los españoles y el indio Berú. El capítulo IV del libro primero describe no solo cómo ocurrió dicha incomunicación, sino también de qué forma los españoles "corrompieron" la lengua general de los incas?'. Paul Firbas describe este hecho como la «escena fundacional de la incomprensión de las asimetrías», pues en ella «[el indio] ofrece con su lengua la materia sonora que los españoles transforman en una palabra de la conquista, borrando el yo y el aquí del indígena»². En tal sentido, los españoles no solo allanaron las complejidades del mundo que ocupaban, sino también satisficieron su deseo de comprender y ser comprendidos: estas

1. Rodolfo Cerrón-Palomino sostiene, a partir de la revisión de los textos de Garcilaso y, particularmente, el cantar de Pachacutiy Inca Yupanqui recogida por el cronista Juan Díez de Betanzos, que los incas utilizaron una lengua particular, el puquina, que era de uso exclusivo de los miembros de la realeza incaica. Esta lengua entró en desintegración debido a la aimarización y/o quechuización de sus hablantes (2013a, p. 59). Para el caso de este trabajo, con lengua general nos referimos al quechua, el cual fue utilizado durante la expansión para afianzar la comunicación entre las poblaciones que conformaron el imperio.

2. Firbas, 2004, p. 277. Además, si seguimos la constitución de las palabras "Cotoche" y "Yucatán", se observa, una vez más, la "corrupción" que se hace de los vocablos "cotohe" y "tectetán" para llegar a estas, respectivamente. 
acciones violentaron la identidad del indio. En el mismo camino, Alberto Escobar entiende que a partir de esta escena, y «en distintos pasajes de los Comentarios, el autor insiste en subrayar, por encima de hechos independientes que absorben el interés del relato, un constante problema de 'comunicación', entendido como fenómeno lingüístico» ${ }^{3}$. Es decir, resulta palpable releer qué relaciones se constituyen en una situación de incomunicación.

En adición a lo anterior, sostengo que la historia de Pedro Serrano, que figura en el capítulo VIII del libro primero, esboza una "verdad poética", aquella que, según Víctor Frankl, se constituye como «una realidad espiritual oculta a los ojos de los hombres vulgares y carnales, y accesible solamente a los hombres dotados de una visión 'poética', a saber, la realidad de los valores ideales que orientan las acciones de un héroe, apareciendo en esta concepción como descubridor de esta 'verdad'»4. En tal sentido, a través del estudio de las fábulas clásicas y renacentistas en los Comentarios reales, Efraín Kristal recuerda que la fábula no es ficción, sino uso de la alegoría para enmascarar una verdad accesible a hombres dotados de una visión poética ${ }^{5}$. Justamente en el texto de León Hebreo, a quien Garcilaso tradujo Los tres diálogos de amor, se afirma que existían tres formas de entender una fábula a través de la metáfora del pelado de una fruta: sentido literal-corteza exterior, sentido moral-corteza intrínseca, sentido alegórico-fruta, médula ${ }^{6}$. En efecto, como lo conciben Frankl y Kristal, la fábula es un relato que contiene verdades alegorizadas; es decir, es una extensión de la verdad o una verdad poética, y el relato de Serrano, desde nuestra lectura, cumple dicha función.

Para Estrella Guerra, la inserción de ficciones dentro de un texto histórico era permitida porque, para la época del Inca, «la función principal de un texto histórico no consistía en dejar un registro lo más fidedigno posible de lo ocurrido, sino que su función primordial era la de educar a partir de lo efectivamente acaecido, para que de ese modo más efectivo» ${ }^{7}$. Ella afirma que el lenguaje y la religión son reforzados en esta fábula, lo que se alinea al objetivo de su obra, que es considerar al Imperio incaico como un antecedente civilizatorio.

En ese camino, Andrea López Salas, siguiendo la nomenclatura que el Inca asignó a la fábula, denomina a esta fábula historial: «aunque a primera instancia los términos [fábula] e [historia] pueden parecer contradictorios, pues parecen enfrentar dos categorías distintas de significación; es decir, la fábula en cuanto relato ficcional y la historia, como referencia a un hecho concreto y verificable, sí es posible enlazarlos en un mismo concepto» 8 . La alegoría que esconde esta fábula historial es, desde el punto de vista de la autora, «preparar al lector al lector para confrontarlo posteriormente con una perspectiva del Imperio incaico que busca reivindicar la

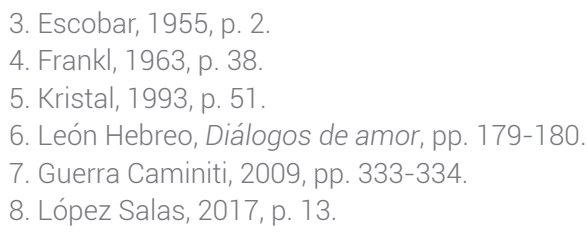


figura del nativo americano» ${ }^{9}$, por lo que el Inca recurre a los conceptos neoplatónicos de alma inmortal y el amor que permiten releer el encuentro cultural dentro de una empresa providencialista. Giovanna Arias Carbone insiste en la misma idea y propone que el Inca Garcilaso tradujo el texto de León de Hebreo de forma meditada como ejercicio filológico y como fundamento ideológico ${ }^{10}$. De esta forma, basado en la corriente neoplatónica de interrelación entre las partes y el todo, sostiene que Garcilaso reelabora los mitos incaicos, las fábulas historiales, para insertarlos en un macrocosmos que tiene al Dios cristiano como centro ${ }^{11}$.

Si bien es cierto que existen autores como Margarita Zamora, quien señala que la reflexión del nombre Perú sirve como una demostración inicial del método filológico y modo de argumentación en los Comentarios reales ${ }^{12}$, y Mark Thurner, quien sostiene que tanto el español Pedro Serrano como el indio Berú representan en la obra del Inca el abismo fundacional que «conecta imperios y funda historias» ${ }^{13}, \mathrm{mi}$ lectura indaga por una comprensión del rol de la lengua en la relación que se entabla con el otro ${ }^{14}$. Como sostiene Escobar, es «como si [se] vislumbrara en la lengua un ingreso natural hacia la realidad» 15 ; es decir, Garcilaso es consciente del conocimiento de la lengua como acceso a la realidad que se describe y, por ello, es importante "aclarar" los yerros de los cronistas anteriores a él cuando dan cuenta de la narración histórica y cuando traducen algunos vocablos del quechua al español.

Ante lo expuesto, considero que la ubicación del relato de Serrano dentro del libro no es gratuita. Efraín Kristal encuentra que las «fábulas [de Garcilaso] no son el producto de una imaginación libre de intenciones extraliterarias. Su significado reside, más bien, en su intención moral y teológica» ${ }^{16}$. Después de hablar de la deducción del nombre Perú y otras deducciones de nombres nuevos, donde expone la "corrupción lingüística" y demuestra su destreza en el dominio del quechua, se inscribe esta historia que expone un problema de comunicación que se resuelve a través de la lengua. Este relato trata sobre las peripecias que sufre Pedro Serrano para sobrevivir en una isla durante siete años. Además de comer mariscos, cangrejos e incluso tortugas crudas, tuvo que lidiar con la falta de agua y de elementos que le ayuden a crear fuego. Después de tres años de estar solo, conoce a un reciente náufrago, quien se acercó a la isla donde estaba Pedro Serrano a causa del humo del fuego que este había logrado sostener. En un inicio, ambos se asustan porque piensan que el otro es la manifestación del demonio: el reciente náufrago observa a Pedro lleno de cabellos y barbas, lo que aleja su apariencia a la de un sujeto dentro de la civilización; por el contrario, Pedro Serrano cree que el demonio se

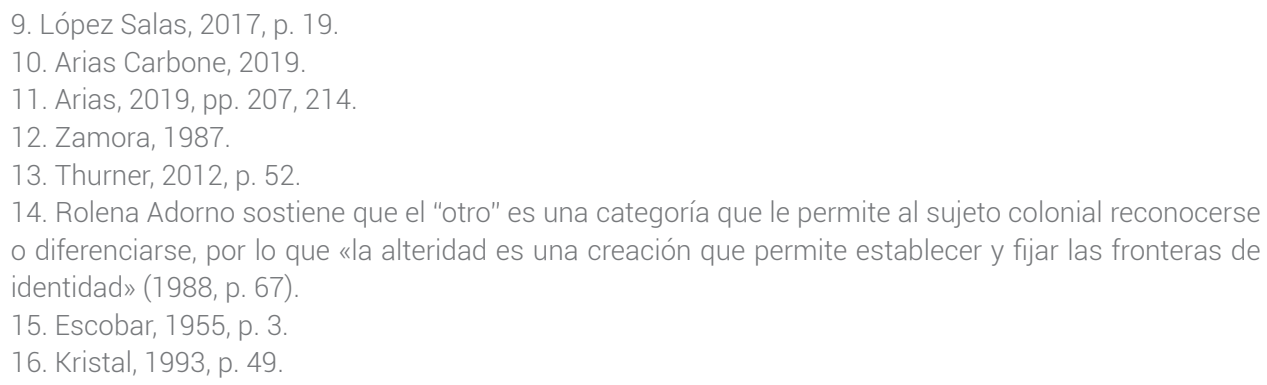


está manifestando en la figura de un hombre para hacerle algún daño; no obstante, a causa de que ambos proclaman a Dios mientras están huyendo, se percatan de que ambos son humanos y se unen para sobrevivir a las adversidades que implica habitar una isla desierta. El relato culmina con el rescate de Pedro Serrano, pues su compañero muere en el camino, su llegada a Alemania para exponer su historia ante el emperador y su viaje a Panamá, donde muere finalmente.

La fábula que acabamos de resumir alude a un problema de comunicación que permite, según nuestra lectura, demostrar que la comprensión de la lengua resulta vital en la relación con el otro. A partir del grito de socorro que Serrano emite en su encuentro con otro hombre, quien «la noche antes se había perdido en los bajos de ella [la isla] y se había sustentado en una tabla del navío» ${ }^{17}$, la lengua se constituye como elemento relacional, pues permite la comprensión y aceptación entre ambos sujetos $^{18}$. Carmela Zanelli menciona al respecto «el relato del náufrago Pedro Serrano, contado para que no quede tan corto el capítulo VIII, pero que encierra, cual fábula que es, metáfora de la relatividad de conceptos tales como los de civilización y barbarie, reconociéndose los náufragos del relato por poseer una religión en común, identificable solo por compartir una lengua» ${ }^{19}$. A partir de la cita queda claro que Serrano se rencuentra con la civilización, la cual está representada en el reciente náufrago, y que el temor de ambos se debe a la interpretación que cada uno realiza según sus apariencias. En el mismo sentido, Domingo Ledezma sostiene que «Pedro Serrano es una metáfora viva de que lo salvaje es una consideración engañosa y superficial que depende de la subjetividad de quien juzga basado meramente en una perspectiva particular, ventajosa o de poder ${ }^{20}$. Por eso, la interpretación errónea basada en los semblantes es superada gracias al diálogo que se entabla entre ambos sujetos: la religión y la lengua consiguen la relación. Así, se transita del temor al afecto, del recelo a la empatía porque la lengua ocupa el lugar común que relaciona a dos sujetos que en un primer momento se veían diferenciados. Al final del relato, ambos logran salir de la isla, pero solo Pedro Serrano sobrevive. No obstante, queda claro que el relato propone una visión opuesta al encuentro de los españoles con el indio Berú. En esta segunda escena, no se violenta la identidad del otro; por el contrario, a partir de la identificación y comprensión, se logra a través de la lengua, se instaura una relación con él. Así, esta es tomada como un elemento de gran importancia porque permite el reconocimiento del otro, paso inicial para establecer relaciones entre los sujetos.

Además de lo expuesto, Pedro Serrano se encuentra apartado de la historia oficial, pues parece destinado a morir en el naufragio; en tal sentido, como sostiene

17. Garcilaso, Comentarios reales, I, VIII, p. 28. Se han modernizado las grafías de todas las citas correspondiente los Comentarios reales.

18. Carlos Garatea aborda los conceptos renacentistas de la lengua en el contexto del Inca Garcilaso. Dentro de ello incide en cómo el Inca resalta su bilingüismo para penetrar en la historia andina. Así, acceder a una lengua andina «permite acceder a una verdad que se escapa a los historiadores por no saber quechua» (2015, p. 259). La lengua resulta, por lo tanto, una herramienta necesaria para acceder al conocimiento del otro.

19. Zanelli, 2016, pp. 425-426.

20. Ledezma, 2010, p. 33 
Trinidad Barrera, «[se abre una] distancia histórica al interrumpirse su continuo vital, su separación de la historia a la que solo se reintegrará en la segunda parte del relato del Inca -haciéndolo desde la marginalidad que su aspecto físico le proporciona $-»^{21}$. Sobre esta lectura, comprender la lengua del otro también resulta vital para la inserción de Pedro Serrano en la línea historial. Si bien es cierto que en un primer momento se establece una distancia geográfica que muestra no solo la lejanía con la civilización, sino también con la naturaleza agreste que no permite desembarcar con facilidad a los navíos, esta se supera porque Serrano es reconocido a pesar de estar cubierto de cabellos, barbas y pelajes. En ese aspecto, la lengua también se constituye como el elemento conector de la identidad individual (Pedro Serrano) con la colectividad (el otro náufrago y quienes rescatan a Pedro Serrano); incluso, su experiencia de vida resulta importante para que Garcilaso lo registre en su proyecto histórico. La lengua, así, le otorga identidad dentro de una colectividad y permite su incorporación a la memoria histórica.

\section{LAS FUNCIONES DEL QUECHUA EN LOS COMENTARIOS REALES}

Con relación a la función de la lengua en el Imperio incaico, Aurelio Miró Quesada sostiene que «es el Inca Garcilaso el primero que reflexivamente analiza el idioma, no para predicar ni gobernar, sino para esclarecer la verdad de los hechos históricos, determinar las áreas geográficas exactas e interpretar la estructura social de sus antepasados por la rama materna»22. Vale decir, Garcilaso indaga sobre el uso y la función del idioma quechua dentro del gobierno incaico y con ello busca entender las relaciones que ejercía dentro de este. En la misma ruta, Alberto Escobar encuentra en el Inca Garcilaso una «anticipada intuición de lingüista moderno», ya que es capaz de presentir «el tema de la comunicación y la realidad historiable; la situación humana de los personajes y el contexto lingüístico vital» ${ }^{23}$. Y no solo ello, Rodolfo Cerrón-Palomino añade que Garcilaso posee una conciencia idiomática que le permite realizar una defensa de las «deturpaciones idiomáticas que... cometían a cada paso los españoles, y los historiadores de su tiempo en particular, no importándoles la integridad de la lengua ni en forma ni en significado» ${ }^{24}$.

Frente a lo expuesto, no sorprende que el Inca reconozca una serie de funciones de la lingua franca en la expansión del Imperio incaico durante el gobierno del Inca Yupanqui, más aún si, como sostiene Roberto González Echevarría, veía a los incas «con una deferencia que en el Renacimiento solía reservarse para sus contrapartidas romanas» ${ }^{25}$. Efectivamente, Amalia Iniesta Cámara encuentra que uno de los proyectos en la obra del Inca es construir una patria imperial incaica. Para ello, a partir de los postulados de Luis E. Valcárcel, sostiene que «el lenguaje [se concibe] como expresión de cultura y como signo de esencias nacionales» y, por lo tanto,

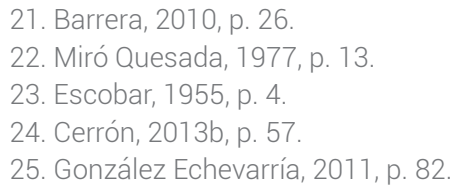


«la lengua fija una personalidad nacional, e interviene por ello en su definición histórica. Recordemos que la patria es la lengua a partir del humanismo; por tanto, el quechua constituye para el Inca Garcilaso la patria imperial de los Incas»²6.

Los siguientes párrafos pretenden enumerar las funciones que cumplió la lengua general durante el gobierno de los Incas y de qué forma otorgó la cohesión social necesaria que aglutinó las diferencias culturales.

En primer lugar, en el capítulo I del libro séptimo, el Inca cuenta cómo el mítmac, que significa indio trocado que migra a otro espacio, era colocado en las nuevas provincias para influir las costumbres y difundir la lengua del imperio. Sin embargo, también el indio conquistado era enviado a la corte para que aprenda la lengua. Así, enfocándonos en la función política, los hijos de los vasallos de los lugares conquistados que todavía no heredaban los Estados eran enviados a la corte para que aprendiesen sus costumbres. Al respecto, el Inca menciona lo siguiente: «los herederos de los señores de vasallos [eran enviados a que] se criasen en la corte y residiesen en ella mientras no heredasen sus estados, para que fuesen bien doctrinados y se hiciesen a la condición y costumbres de los Incas, tratando con ellos amigablemente, para que después, por la comunicación y familiaridad pasada, los amasen y sirviesen con afición ${ }^{27}$. Como se expresa en la cita, el fin de esta imposición era entablar un lazo de familiaridad con las autoridades de las provincias, todo ello con el propósito de afianzar la conquista y ganar terreno en la población anexada. Los incas eran conscientes de que, si la cabeza del pueblo conquistado se sometía a sus costumbres, los demás pobladores, por imitación, envidia o respeto, harían lo mismo. En efecto, Garcilaso sostiene que «causaban grande envidia para que los demás la deseasen y procurasen saber, y los que así sabían algo, por pasar adelante en el lenguaje, trataban más a menudo y más familiarmente con los gobernadores y ministros de la justicia y de la hacienda real, que asistían en sus tierras» ${ }^{28}$.

En segundo lugar, los incas usaban esta migración como prevención bélica, ya que al lograr esta confederación filial consiguen proteger las provincias conquistadas de posibles levantamientos o rebeliones. En tal sentido, el mítmac que venía de lugares lejanos era mejor tratado en relación con los que estaban más cerca de la Corte, pues los incas entregaban regalos y favores para refrenar sus deseos de liberación y para que estuviesen agradecidos con ellos. Al respecto, Garcilaso expone cómo los pueblos no conquistados por los Incas entraban en batalla unos con otros porque no tenían una lengua común con que comunicarse: «Los que se entendían en un lenguaje se tenían por parientes, y así eran amigos y confederados. Los que no se entendían, por la variedad de las lenguas, se tenían por enemigos y contrarios, y se hacían cruel guerra, hasta comerse unos a otros como si fueran brutos de diversas especies» ${ }^{29}$.

26. Iniesta Cámara, 2010, p. 157.

27. Garcilaso, Comentarios reales, VII, II, p. 89.

28. Garcilaso, Comentarios reales, VII, II, p. 90.

29. Garcilaso, Comentarios reales, I, XIV, p. 38 
En tercer lugar, la imposición de la lengua cumplía una función pedagógica. Ante la proliferación de distintas lenguas en todo el Perú, los Incas optaron por imponer el quechua con el fin de no tener gran cantidad de intérpretes. En este aspecto, Garcilaso recurre al padre Valera para corroborar la facilidad de la evangelización gracias a la imposición de la lingua franca, ya que no era necesario aprender todas las lenguas de las naciones o provincias del Perú, sino que bastó conocer el quechua para iniciar la prédica del cristianismo ${ }^{30}$.

En cuarto lugar, y siguiendo la idea anterior, el idioma no solo facilitó la comunicación entre los individuos, sino también desarrolló la capacidad e ingenio de quienes la aprenden. Así, el padre Valera cree que «les hace más agudos de entendimiento y más dóciles y más ingeniosos para lo que quisieren aprender, y de bárbaros los trueca en hombres políticos y más urbanos [...] cuando alcanzan a saber la lengua del Cuzco paresce que echan de sí la rudeza y torpeza que tenían y que aspiran a cosas políticas y cortesanas y sus ingenios pretenden subir a cosas más altas» ${ }^{31}$. Justamente, este desarrollo del entendimiento a través del idioma constituye una praeparatio evangelica, pues los evangelizadores encuentran a los indios más dispuestos para recibir el cristianismo gracias al ordenamiento y desarrollo que el Imperio incaico logró en la cultura y costumbres de los habitantes del Perú.

En quinto lugar, poseer una misma lengua facilitó la relación económica y comercial entre las distintas provincias del imperio. En tal sentido, el Inca anota lo siguiente: «la lengua cortesana tiene este don particular, digno de ser celebrado, que a los indios del Perú les es de tanto provecho como a nosotros la lengua latina; porque demás del provecho que les causa en sus comercios, tratos y contratos.... ${ }^{32}$. La escasez de un producto por la geografía o el clima obligaba a las provincias a intercambiar alimentos. A media que se conquistaban poblaciones, los climas fríos o templados permitían cultivar productos como el maíz, papa, quinua, incluso criar el ganado. Mantener un mismo idioma en toda la población facilitó el intercambio de estos productos.

En sexto lugar, y ante todo lo expuesto, la imposición de la lengua permitió la cohesión social de todos los habitantes del Imperio incaico. Garcilaso comprendía que la lengua era un elemento de enlace vital en la construcción del imperio, y que la "corrupción" que los españoles hicieron de la lengua alteraba drásticamente la comprensión de su cultura. Al respecto, Estrella Guerra sostiene que «la principal característica del hombre sabio que educa al resto es su manejo del lenguaje. La palabra se identifica con la racionalidad, lo cual era también uno de los tópicos de la época; lo que diferencia al hombre bárbaro del hombre educado es su uso del lenguaje» ${ }^{33}$. Este despliegue del impero por considerar a la lengua una muestra de raciocinio ayuda, de nuevo, al objetivo del libro de considerar que los incas prepararon las condiciones para la inserción del cristianismo y, por lo tanto, ambos compartían un Dios único. Garcilaso representa a los incas como el imperio digno

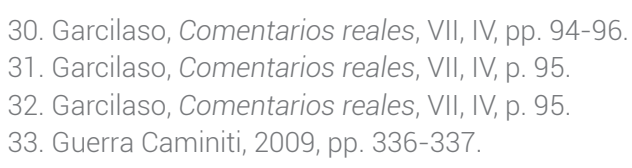


de ser registrado en la historia universal, el cual se encuentra en el mismo nivel cultural de Roma o Grecia. En efecto, tanto las lecturas de Efraín Kristal, desde la inclusión del relato de los gentiles como prefiguración cristiana que se realizó en el Imperio romano a través de verdades ocultas que pueden ser descifradas, pues son alegoría ${ }^{34}$, como Carlos Garetea, quien se centra en el símil que realiza el Inca entre Cuzco y Roma como centros culturales y, por extensión, a patrones lingüísticos de prestigio ${ }^{35}$, afirman la intención de Garcilaso de incluir al Imperio incaico dentro de la historia universal.

De hecho, América era vista como un paraíso occidental, pues en ella los pobladores desconocían la propiedad privada, según sostenía el Padre Acosta. Al respecto, Joaquim Mallafré Gavaldá expresa que «los indios tenían unos elementos utópicos suyos y los europeos trataron de aplicar los propios, a veces conflictivos, al creer ver allí su propio concepto de utopía» ${ }^{36}$.

Finalmente, si seguimos a Margarita Zamora, quien cree que «Garcilaso se propone no solo una traducción correctiva, sino un método hermenéutico que le permitirá acercarse al sepultado y fragmentario texto original de la historia incaica para reintegrarlo al canon de la historiografía europea sobre la América prehispánica» ${ }^{37}$, es posible entender que la distintas funciones que otorga el Inca Garcilaso a la lengua de la corte incaica sirven para afianzar esta exaltación del Imperio incaico. Así, como sostiene Michael Karl Schuessler, «para Garcilaso, la escritura europea no solamente se compara con los sistemas elaborados por los incas, sino que actúa como un agente que reduce y tergiversa la tradición de su cultura en vez de ampliar y revelar su admirable trayectoria» 38 , la propuesta es restaurar las "tergiversaciones" lingüísticas que los españoles hicieron del idioma quechua y revalorar la función que cumplió la lengua en la construcción de su imperio.

\section{CONCLUSIONES}

La reflexión sobre la función de la lengua a lo largo de la primera parte de los Comentarios reales busca resaltar la importancia de esta en la construcción identitaria y en el conocimiento de la cultura. Por un lado, como sostiene José Durand, el Inca llena la historia de virtudes poéticas, no solo para romper la monotonía de la historia política, sino también para construir verdades poéticas ${ }^{39}$. En el caso de la historia de Pedro Serrano, esta expresa la importancia de la lengua como elemento relacional que permite entablar un vínculo con el otro. Esto se afianza cuando el relato se ubica inmediatamente después de describir la "corrupción y alteraciones" que los españoles hicieron de los vocablos quechuas. Esto último ocurre con el fin de imponer su visión y borrar, a través de un nombre general, las complejidades de

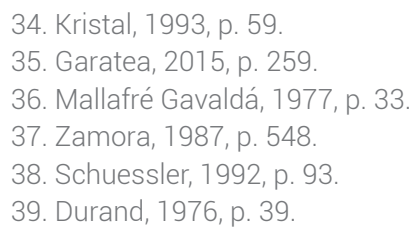


los indios, justo antes de iniciar su relato sobre la historia del Perú y antes de presentar a los "salvajes" pueblos preincas.

Por otro lado, el Inca Garcilaso colecciona los distintos usos y funciones que cumplió la lengua quechua en la constitución y expansión del Imperio incaico. La imposición de una sola lengua permitió no solo transmitir más fácilmente las tradiciones y costumbres de los Incas en los pueblos conquistados, sino también dinamizar las relaciones económicas y comerciales del imperio. Además, Garcilaso está a favor de la propuesta del Padre Valera, quien sostiene que la lengua quechua desarrolló el entendimiento de los indios y los preparó para enriquecer su ingenio y la conversión cristiana.

De esta forma, tanto en la escena inicial de la incomprensión lingüística entre los españoles y el indio Berú, y en la posibilidad de diálogo entre Pedro Serrano y el otro náufrago, como en la constitución de la lengua quechua como ensamblaje vital del Imperio incaico, se observa una conciencia idiomática que trasciende las correcciones lingüísticas. La importancia de la lengua no solo radica en ser síntoma de una sociedad civilizada; también, apunta a indagar la verdad a través de la reconstrucción de la realidad histórica mediante la relación con el otro. El Inca, en su doble identidad de español e indio, y gracias al conocimiento del español y el quechua, apuesta por el redescubrimiento de su cultura, aquella que ha sido mal interpretada y corrompida por la incompetencia lingüística de los cronistas españoles. Se constituye, como apunta Zanelli, en «el preámbulo perfecto para iniciar a relatar su historia del Perú y de los Incas, a quienes no se les ha entendido en las crónicas españolas y a quienes debe servir, entonces, de "comento y glosa"»40. Recordemos, por el contrario, su objetivo en la primera parte: «Yo, incitado del deseo de la conservación de las antiguallas de mi patria, esas pocas que han quedado, por que no se pierdan del todo, me dispuse al trabajo tan excesivo como hasta aquí me ha sido y delante me ha de ser, al escribir su antigua república hasta acabarla» ${ }^{41}$.

\section{BiBLIOgRAFÍA}

Adorno, Rolena, «El sujeto colonial y la construcción cultural de la alteridad», Revista de Crítica Literaria Latinoamericana, 28, 1988, pp. 56-68.

Arias Carbone, Giovanna, «Tan diversas y altas sentencias": neoplatonismo y preparación evangélica en los Comentarios reales de los Incas», Hipogrifo. Revista de literatura y cultura del Siglo de Oro, 7.2, 2019, pp. 201-216.

Barrera, Trinidad, «Otra vuelta de tuerca al naufragio de Pedro Serrano», en Renacimiento mestizo: los 400 años de los «Comentarios reales», ed. José Antonio Mazzotti, Madrid / Frankfurt am Main, Iberoamericana / Vervuert, 2010, pp. 19-29. 
Cerrón-Palomino, Rodolfo, «El Inca Garcilaso o la lealtad idiomática», en Tras las huellas del Inca Garcilaso. El lenguaje como hermenéutica en la comprensión del pasado, Boston, Latinoamericana Editores / CELACP / Revista de Crítica Literaria Latinoamericana, 2013a, pp. 27-78.

Cerrón-Palomino, Rodolfo, «La lengua particular de los incas», en Las lenguas de los incas: el puquina, el aimara y el quechua, Frankfurt, PL Academic Research, 2013b, pp. 53-82.

Durand, José, «Garcilaso el Inca, platónico», en El Inca Garcilaso, clásico de América. México, SepSetentas, 1976, pp. 32-46.

Escobar, Alberto, «Lenguaje e historia en los Comentarios reales», en Patio de Letras 3, Lima, Luis Alfredo Ediciones, 1955, pp. 1-22.

Firbas, Paul, «La geografía antártica y el nombre del Perú», en La formación de la cultura virreinal. II. El siglo XVIII, ed. Karl Kohut y Sonia V. Rose, Madrid / Frankfurt am Main, Iberoamericana / Vervuert, 2004, pp. 265-287.

Frankl, Víctor, El «Antijovio» de Gonzalo Jiménez de Quesada y las concepciones de realidad y verdad en la época de la contrarreforma y del manierismo, Madrid, Ediciones de Cultura Hispánica, 1963.

Garatea, Carlos, «La idea de lengua en los Comentarios reales del Inca Garcilaso de la Vega», Romanistisches Jahrbuch, 66.1, 2015, pp. 256-276.

Garcilaso de la Vega, Inca, Comentarios reales de los Incas [1609], ed. Ángel Rosenblat, Buenos Aires, Emecé Editores, 1943, 2 vols.

González Echevarría, Roberto, «La ley de la letra: Ios Comentarios de Garcilaso», en Mito y archivo. Una teoría de la narrativa latinoamericana, México, Fondo de Cultura Económica, 2011, pp. 80-143.

Guerra Caminiti, Estrella, «La digresión en los Comentarios Reales de los Incas: ¿oportunidad para la ficción? El caso de Pedro Serrano», Lexis, 33, 2009, pp. 21-35.

Iniesta Cámara, Amalia, «La construcción de una patria andina a través de la escritura de los Comentarios reales», en Humanismo, mestizaje y escritura en los «Comentarios reales», ed. Carmen de Mora, Guillermo Serés y Mercedes Serna, Madrid / Frankfurt am Main, Iberoamericana / Vervuert, 2010, pp. 149-168.

Hebreo, León, Diálogos de amor de León Hebreo, trad. del Inca Garcilaso de la Vega, ed. Eduardo Juliá Martínez, Madrid, Librería General Victoriano Suárez, 1949

Kristal, Efraín, «Fábulas clásicas y neoplatónicas en los Comentarios reales de los incas», en Homenaje a José Durand, ed. Luis Cortest, Madrid, Editorial Verbum, 1993, pp. 47-59. 
Ledezma, Domingo, «Los infortunios de Pedro Serrano: huellas historiográficas de un relato de naufragio», en Renacimiento mestizo: los 400 años de los «Comentarios reales», ed. José Antonio Mazzotti, Madrid / Frankfurt am Main, Iberoamericana / Vervuert, 2010, pp. 31-50.

López Salas, Andrea Vanesa, Entre un «caso historial de grande admiración» y un relato ficcional: el episodio de Pedro Serrano como alegoría de la conquista en los «Comentarios Reales» del Inca Garcilaso, Tesis para optar el grado de Licenciada en Lingüística y Literatura con mención en Literatura Hispánica, Lima, Pontificia Universidad Católica del Perú, 2017.

Mallafré Gavaldá, Joaquim, «Las utopías posteriores», en Tomás Moro, Utopía, Barcelona, Bosch, 1977, pp. 53-57.

Miró Quesada, Aurelio, «Las ideas lingüísticas del Inca Garcilaso», en Tiempo de leer, tiempo de escribir, Lima, Talleres Gráficos P. L. Villanueva S.A., 1977, pp. 11-49.

Schuessler, Michael, «Garcilaso escribe "como indio". El concepto y la función de la escritura incaica en los Comentarios reales del Inca Garcilaso de la Vega», Mester, 21.2, 1992, pp. 83-96.

Thurner, Mark, «Capítulo 1. El abismo fundacional», en El nombre del abismo. Meditaciones sobre la historia de la historia, Lima, Instituto de Estudios Peruanos, 2012, pp. 19-52.

Zamora, Margarita, «Filología humanista e historia indígena en los Comentarios reales», Revista Iberoamericana, 140, 1987, pp. 547-558.

Zanelli, Carmela, «Las fábulas de Garcilaso: alegoría, historia o ficción en los Comentarios reales», Lexis, 40.2, 2016, pp. 421-433. 